\title{
Lung transplantation: chronic rejection and stem cell depletion
}

\author{
Thomas J. Lynch, Bethany A. Ahlers, Kalpaj R. Parekh \\ Division of Cardiothoracic Surgery, Department of Surgery, Carver College of Medicine, University of Iowa, Iowa City, IA, USA \\ Correspondence to: Kalpaj R. Parekh, MBBS. Director, Division of Cardiothoracic Surgery, Department of Surgery, University of Iowa Hospitals and \\ Clinics, 200 Hawkins Drive SE 514GH, Iowa City, IA 52242, USA. Email: kalpaj-parekh@uiowa.edu. \\ Provenance: This is an invited article commissioned by Dr. Prasad S. Adusumilli (Thoracic Service, Department of Surgery, Memorial Sloan Kettering \\ Cancer Center, New York, USA). \\ Response to: Xian W, McKeon F. Demise of lung transplants: exposing critical gaps in understanding lung stem cells. J Thorac Dis 2018;10:S1016-9.
}

Submitted Jun 30, 2018. Accepted for publication Jul 12, 2018.

doi: $10.21037 /$ jtd.2018.07.70

View this article at: http://dx.doi.org/10.21037/jtd.2018.07.70

Chronic lung diseases are a major global health concernaccounting for approximately $13.5 \%$ of all deaths worldwide (1). Lung transplantation is an effective therapy for end-stage lung diseases when medical therapies fail. While lung transplantation does provide an excellent quality of life, unfortunately, a majority of lung-transplant recipients develop obliterative bronchiolitis $(\mathrm{OB})$ within 5 years of transplantation, significantly reducing the long-term survival prospects of these patients (2). The reasons why lung-transplant recipients develop OB is not entirely understood, but it is believed to result in part from injury-induced exhaustion of airway epithelial stem cells (3-5). Our recent findings confirm that as chronic lung allograft disease pathology worsens somatic lung stem cells diminish from both small and large airways and submucosal glands (SMGs) (5). Understanding the mechanisms that contribute to stem cell depletion will enable further interventions aimed at treating and/or preventing OB.

Inflammation and irreversible fibrosis in the OB lung obstructs airflow in small airways and eventually causes respiratory failure and patient death. These findings led to the paradigm that $\mathrm{OB}$ pathobiology is confined to small airways. However, recent ferret and human histological studies in our laboratory indicate that this may not be entirely the case, and some prominent histological changes in large airways may actually precede small airway occlusion (5). Specifically, we have now shown that there is a significant loss of large airway SMGs and a severe decline in $\mathrm{K}^{+} \mathrm{p} 63^{+}$basal cells within both large and small airways preceding small airway lesions characteristic of OB (5).
Clinically, recognition of the fact that phenotypic changes associated with chronic rejection occur early in disease progression and in large airways that are readily accessible by bronchoscopy may have profound implications for earlier diagnosis and may enable more effective interventions in OB by identifying a specific cell population for therapeutic targeting.

From a broader perspective on regenerative biology in the lung the observation that multiple epithelial stem cell compartments are depleted during chronic injury suggests that there is a significant interrelationship between stem cells of distinct levels of the conducting airway. Ample evidence exists that epithelial regeneration/repair in the adult lung is context-specific and can engage multiple stem cell populations sometimes involving cell fate plasticity (i.e., non-linear lineage relationships) (6-10). However, there remains much to be discovered. For example, epithelial repair processes may change over the course of pathoprogression. In chronic rejection and $\mathrm{OB}$, we do not yet fully understand how $\mathrm{K}^{+} \mathrm{p} 63^{+}$basal cells are replaced with $\mathrm{K}^{4} 4^{+}$basal cells in large and small airways and how this impacts lung regeneration. Additionally, SMG loss may represent a pivotal early step in OB progression because recent lineage trace analysis of glandular myoepithelial cells (MECs) in mice revealed that MECs are not only capable of regenerating glandular cell types but are also capable of serving as reserve stem cells for the regeneration of surface airway epithelial cell types including multipotent surface airway basal cells $(6,10)$. In addition, glandular stem cells (perhaps MECs) but not surface airway basal cells have the unique ability to generate gland-like clones 
in denuded tracheal xenografts further emphasizing their potential importance for maintaining lasting basal stem cell populations $(6,11)$. Given that SMGs are destroyed prior to end-stage OB (5), we hypothesize that MECs have a critically important lineage relationship with surface airway epithelial basal cells in the face of chronic injury. Therefore, we aim to better understand the driving mechanisms that lead to SMG destruction as well as surface airway stem cell depletion in lung transplantation.

When studying stem cells in the lung, it may be important to note that there are considerable differences between humans and readily available rodent models. For example, human SMGs extend throughout cartilaginous airways, whereas mouse SMGs are confined to the proximal trachea. In addition, basal stem cells extend further down the respiratory tree and are abundant in distal airways of humans, while murine lungs have far fewer distal airway basal cells. Ferrets are highly similar to humans in regard to SMG and distal airway basal cell abundance and distribution. Thus, as illustrated in our studies of ferret and human lung transplantation, the ferret model may help elucidate critically important mechanisms that control stem cell dynamics in humans but may be less salient in rodents.

Whether the destruction of SMG MECs is ultimately responsible for depletion of basal stem cells in the proximal and distal airways in $\mathrm{OB}$ remains to be determined. Nevertheless, our data indicates that stem cell therapy following lung-transplantation may offset the disruption of endogenous airway stem cell niches and prolong patient survival. Therefore, further studies utilizing the ferret lung transplant model to develop methods for autologous stem cell engraftment is merited. We believe the key to optimizing lung transplant success lies within the regeneration of surface airway epithelium (SAE) and SMG stem cells possibly through stem cell therapy, and knowledge gained from interrogating stem cell therapy in the ferret $\mathrm{OB}$ model may translate broadly to other lung disease contexts.

However, several challenges remain before stem cell therapy can become a broadly applicable treatment option for lung diseases. For example, one key challenge is to identify a distinct endogenous cell type or mixture of cell types that will yield maximal efficacy for lasting engraftment and regeneration in the lung. Further, the ability to rapidly expand and deliver these cells to the appropriate compartments within the damaged or diseased lung will need optimization using animal models. In addition, efficient gene correction/editing of endogenous stem cells may be necessary prior to therapeutic delivery to effectively treat genetic diseases such as cystic fibrosis. Finally, the ability of therapeutic stem cells to provide lasting and functional regeneration in the lungs without malignant transformation must be demonstrated in animal models. Though there is a long road ahead, progress toward developing stem cell therapy as a safe and efficacious treatment option for endstage lung disease is rapidly underway-making stem cell therapy one of the most exciting translational frontiers in biomedical research.

\section{Acknowledgements}

This study was supported by Cystic Fibrosis Foundation Grant (PI: KR Parekh).

\section{Footnote}

Conflicts of Interest: The authors have no conflicts of interest to declare.

\section{References}

1. GBD 2013 Mortality and Causes of Death Collaborators. Global, regional, and national age-sex specific all-cause and cause-specific mortality for 240 causes of death, 19902013: a systematic analysis for the Global Burden of Disease Study 2013. Lancet 2015;385:117-71.

2. Todd JL, Palmer SM. Bronchiolitis obliterans syndrome: the final frontier for lung transplantation. Chest 2011;140:502-8.

3. Gilpin SE, Lung KC, Sato M, et al. Altered progenitor cell and cytokine profiles in bronchiolitis obliterans syndrome. J Heart Lung Transplant 2012;31:222-8.

4. Kelly FL, Kennedy VE, Jain R, Sindhwani NS, Finlen Copeland CA, Snyder LD, Eu JP, Meltzer EB, Brockway BL, Pavlisko E, et al. Epithelial clara cell injury occurs in bronchiolitis obliterans syndrome after human lung transplantation. Am J Transplant 2012;12:3076-84.

5. Swatek AM, Lynch TJ, Crooke AK, et al. Depletion of Airway Submucosal Glands and TP63(+)KRT5(+) Basal Cells in Obliterative Bronchiolitis. Am J Respir Crit Care Med 2018;197:1045-57.

6. Lynch TJ, Anderson PJ, Rotti PG, et al. Submucosal Gland Myoepithelial Cells Are Reserve Stem Cells That Can Regenerate Mouse Tracheal Epithelium. Cell Stem Cell 2018;22:779.

7. Yee M, Domm W, Gelein R, et al. Alternative Progenitor Lineages Regenerate the Adult Lung Depleted of Alveolar 
Epithelial Type 2 Cells. Am J Respir Cell Mol Biol 2017;56:453-64.

8. Tata PR, Rajagopal J. Plasticity in the lung: making and breaking cell identity. Development 2017;144:755-66.

9. Hogan BL, Barkauskas CE, Chapman HA, et al. Repair and regeneration of the respiratory system: complexity, plasticity, and mechanisms of lung stem cell function. Cell Stem Cell 2014;15:123-38.

Cite this article as: Lynch TJ, Ahlers BA, Parekh KR. Lung transplantation: chronic rejection and stem cell depletion. J Thorac Dis 2018;10(8):E666-E668. doi: 10.21037/ jtd.2018.07.70
10. Tata A, Kobayashi Y, Chow RD, et al. Myoepithelial Cells of Submucosal Glands Can Function as Reserve Stem Cells to Regenerate Airways after Injury. Cell Stem Cell 2018;22:668-83.e6.

11. Lynch TJ, Anderson PJ, Xie W, et al. Wnt Signaling Regulates Airway Epithelial Stem Cells in Adult Murine Submucosal Glands. Stem Cells 2016;34:2758-71. 